\title{
Kumar IMWG 2016 Oncology Response Criteria
}

National Cancer Institute

\section{Source}

National Cancer Institute. Kumar IMWG 2016 Oncology Response Criteria. NCI

Thesaurus. Code C147478.

A tumor response criterion published in 2016 by Kumar et al. entitled International Myeloma Working Group consensus criteria for response and minimal residual disease assessment in multiple myeloma. (Kumar S, Paiva B, Anderson KC, Durie B, et al. International Myeloma Working Group consensus criteria for response and minimal residual disease assessment in multiple myeloma. Lancet Oncol. 2016 Aug;17(8):e32846.) 